\title{
Artificial Selection of Gn1a Plays an Important role in Improving Rice Yields Across Different Ecological Regions
}

\author{
Jie Wang, Huaxue Xu, Nengwu Li, Fengfeng Fan, Liuting Wang, Yingguo Zhu and Shaoqing Li ${ }^{*}$
}

\begin{abstract}
Background: Rice is one of the most important crops, and it is essential to improve rice productivity to satisfy the future global food supply demands. Gn1a (OsCKX2), which encodes cytokinin oxidase/dehydrogenase, plays an important role in regulating rice grain yield.

Results: In this study, we analyzed the genetic variation of $G n 1 a$, which influences grain yield through controlling the number of spikelets in rice. The allelic variations in the promoter, $5^{\prime}$ untranslated region (UTR) and coding sequence (CDS) of Gn1a were investigated in 175 cultivars and 21 wild rice accessions. We found that Gn1a showed less sequence variation in the cultivars, but exhibited significant nucleotide diversity in wild rice. A total of 14 alleles, named AP1 to AP14, were identified in the cultivars based on the amino acid divergence of GN1A. Association analysis revealed that the number of spikelets and grain yield were significantly different between the different alleles. Phylogenetic analysis indicated that the three main alleles, AP3, AP8 and AP9, in the cultivars might originate from a common ancestor allele, AP1, in wild rice.
\end{abstract}

Conclusions: Of these alleles in the cultivars, AP9 was suggested as the best allele in indica, as it has shown strong artificial selection in breeding high-yield rice in the past. It might be valuable to explore the high-yield-related alleles of Gnla to develop high-yield rice cultivars in future breeding programs.

Keywords: Rice, Artificial selection, Gn1a, Allele, Association analysis

\section{Background}

With the rapid decrease in the available farmland and increase in the global population, it is urgent to obtain enough grain production to meet the food supply demands (Weng et al. 2008). With more than 10,000 varieties, rice is a safe and staple food for half of the world's population (Miura et al. 2010), and thus improving rice productivity is a significant challenge due to the expansion of the world's population. Rice grain yield is controlled by four basic components, including number of panicles per plant, number of spikelets per panicle, the seed setting rate, and the grain weight, all of which are typical quantitative traits (Xing and Zhang 2010). In the

\footnotetext{
*Correspondence: shaogingli@whu.edu.cn

State Key Laboratory of Hybrid Rice; Key Laboratory for Research and Utilization of Heterosis in Indica Rice of Ministry of Agriculture; Engineering Research Center for Plant Biotechnology and Germplasm Utilization of Ministry of Education; College of Life Science, Wuhan University, Wuhan 430072, China
}

\section{里

last few decades, a large number of QTLs related to rice grain yield, such as Gn1a (Ashikari et al. 2005), OsSPL14 (Jiao et al. 2010; Miura et al. 2010), APO1 (Ikeda et al. 2007), DEP1 (Huang et al. 2009), OsARG (Ma et al. 2012), TAWAWA1 (Yoshida et al. 2013), OsEBS (Dong et al. 2013), DST (Li et al. 2013) and PAY1 (Zhao et al. 2015), had been functionally characterized by positional cloning. These important functional genes are usually generated from mutations that occurred in natural populations, such as wild rice and landraces, the ancestors of modern cultivars. Of these, Gn1a (OsCKX2) is the first gene to be isolated that controls the rice grain yield by regulating cytokinin and the number of spikelets. Reduced expression of Gn1a causes cytokinin accumulation in the inflorescence meristems and, consequently, increases the number of spikelets (Ashikari et al. 2005). Gn1a plays an important role in regulating rice grain yield. However, the allelic diversity and evolutionary relationship of Gn1a in rice populations and the genetic 
effects of these alleles on grain yield are still unclear. Thus, it is important to explore the potentially favorable alleles of Gn1a to improve rice yield when designing strategies in molecular breeding programs.

In this study, the variation and evolutionary pattern of GN1A in wild rice and landraces were investigated. The relationships between the alleles and rice yield components, including the number of spikelets, thousand-grain weight, seed setting rate and effective panicles per plant, were analyzed by association analysis. We found that the high-yield-related alleles of GN1A in the rice populations showed strong artificial selection during rice domestication, which indicated that Gn1a played an important role in rice improvements.

\section{Results}

\section{Nucleotide Variations of $\mathrm{Gn} 1 \mathrm{a}$}

The Gn1a sequences were cloned and sequenced from the cultivar lines and wild rice accessions, which represented a diverse range of spikelet numbers. The 5'UTR and coding frame of $1761 \mathrm{bp}$ were investigated in $175 \mathrm{cul}-$ tivar lines (Additional file 1: Table S1) and 21 wild rice accessions of O. rufipogon (Additional file 2: Table S2). The average number of nucleotide differences per site between any two DNA sequences from the sample population $(\pi)$ was used to estimate the polymorphisms between $O$. sativa and O. rufipogon (Table 1). The nucleotide diversity of Gn1a from the indica and japonica cultivars and $O$. rufipogon were $0.00108,0.00108$ and 0.00203 , respectively. The $\pi$ value of $O$. rufipogon $(0.00203)$ was higher than that of $O$. sativa (0.00110). The sliding-window diagram showed that the majority of the variable sites between $O$. sativa and O. rufipogon were located in exon 2 and exon 3 of Gn1a (Fig. 1). This finding indicated that the Gn1a alleles were more diverse in the wild rice accessions than in the cultivars.

Based on the nucleotide variations, a total of 17 single nucleotide polymorphisms (SNPs) and five insertion/deletions (indels) of Gn1a were detected in O. sativa. These SNPs and indels represented 22 combinations of DNA sequence variations and were named A1 to A22 (allele 1 to allele 22, Table 2). Of these, two indels located at site 664 and 667 caused frameshifts and produced premature GN1A proteins; the other two indels in the CDS caused amino acid deletions. Additionally, two synonymous SNPs occurred at site 741 and 828 in the second exon. Previous research showed that there were three SNPs and three indels in the 5'UTR and coding sequence of Gn1a, including an 11-bp deletion in the third exon, which generated a large panicle in 5150 (Ashikari et al. 2005). The 11-bp deletion in the third exon was not detected in our tested cultivars, but was detected in the wild rice accession, indicating that the 11-bp deletion in the third exon might originate from O. rufipogon. Then, we analyzed the divergence of protein sequence of each allele and found 14 variations in the cultivars at protein level, which were renamed as alleles AP1 to AP14 (alleles for protein 1 to protein 14, Table 3). Among these alleles, AP5, AP10 and AP13 formed premature proteins, and the others only changed the number of amino acids.

\section{Phenotypic Analysis of the GN1A Alleles}

As Gn1a functions to control rice yield by regulating the number of spikelets, we analyzed the relationship between the AP alleles and yield components, including the effective panicles per plant, thousand-grain weight, seed setting rate, and spikelets per panicle. Because alleles AP3, AP8 and AP9 represented the highest frequency, over $10 \%$ in the indica subpopulation (Table 3), they were used for the association analysis. The result showed that there was a significant difference in the effective panicles per plant between AP3 and the other two alleles, but there was no difference between AP8 and AP9 (Fig. 2a). There was a significant difference in the seed setting rate between AP3 and both AP8 and AP9, although no significant difference was detected between the AP8 and AP9 alleles (Fig. 2b). For the spikelets per panicle, the means of AP3, AP8 and AP9 were 119.50, 124 and 144.73, respectively, which showed an extremely significant difference $(P<0.01)$ between AP9 and AP3, and a significant difference $(P<0.05)$ between AP8 and AP9 (Fig. 2c). These results suggested that cultivars with the AP9 allele had a higher number of spikelets per panicle compared to AP3 and AP8, without changing the thousand-grain weight (Fig. 2d). However, there was no significant difference in the grain yield per plant between AP8

Table 1 Polymorphisms and neutrality tests of Gnla in different species

\begin{tabular}{llllll}
\hline Sample population & $\pi$ & $\theta w$ & Tajima's D & Fu and Li's D & Fu and Li's F \\
\hline Oryza sativa $(\mathrm{n}=175)$ & 0.0011 & 0.00162 & -0.85199 & $-3.34175^{*}$ & $-2.86108^{*}$ \\
Oryza rufipogon $(\mathrm{n}=21)$ & 0.00203 & 0.00382 & -1.79223 & -1.27594 & -1.66681 \\
Indica-like accessions $(\mathrm{n}=145)$ & 0.00108 & 0.00168 & -0.96016 & $-3.16946^{*}$ & $-2.79096^{*}$ \\
Japonica-like accessions $(\mathrm{n}=32)$ & 0.00108 & 0.00095 & 0.41135 & 1.26945 & 1.17766 \\
\hline
\end{tabular}

The number in bracket represents the number of corresponding materials. "** indicates a significant difference at $\mathrm{P}<0.05$ 


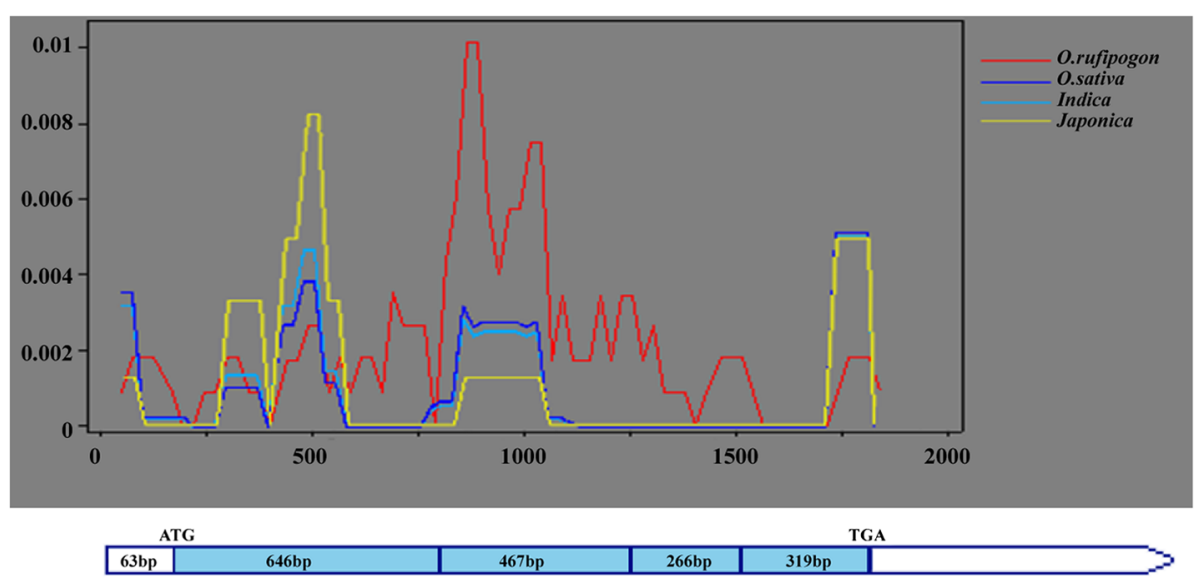

Fig. 1 Sliding-window analysis for the Gnla alleles in O. rufipogon, O. sativa, indica and japonica cultivars. The genomic structure from the 5'UTR to the stop codon (excluding introns) is shown at the bottom; the blue boxes and white boxes indicate the exons and UTRs. The red, blue, light green and yellow curves represent the O. rufipogon, O. sativa, indica cultivars and japonica cultivars, respectively

and AP9 (Fig. 2e) because AP8 had a relatively higher number of panicles per plant than AP9. From the results above, we concluded that AP9 was more prevalent than AP8 in cultivars because it produced more spikelets per panicle.

\section{Expressional Analysis of the Gn1a Alleles}

To determine whether the Gn1a transcript was closely associated with the number of spikelets, we analyzed the promoter and 5'UTR of Gn1a, which contains $1500 \mathrm{bp}$ upstream ATG of Gn1a in the cultivars, and found two

Table 2 Nucleotide variations in the 5'UTR and CDS of Gn1a

\begin{tabular}{|c|c|c|c|c|c|c|c|c|c|c|c|c|c|c|c|c|c|c|c|c|c|c|}
\hline Alleles & -124 & -31 & -7 & 161 & 236 & 319 & 353 & 661 & 663 & 664 & 665 & 666 & 667 & 688 & 741 & 781 & 782 & 828 & 885 & 910 & 984 & 1611 \\
\hline $\mathrm{A} 1$ & $A$ & $A$ & $16 \mathrm{bp}$ & $G$ & $6 \mathrm{bp}$ & $G$ & $A$ & G & $G$ & $A$ & $\mathrm{~T}$ & G & G & G & C & G & C & C & G & G & $3 b p$ & $\mathrm{~T}$ \\
\hline A2 & A & A & $16 \mathrm{bp}$ & C & - & G & G & G & G & A & $\mathrm{T}$ & G & G & G & C & G & C & C & G & G & $3 b p$ & $\mathrm{~T}$ \\
\hline A3 & A & A & $16 \mathrm{bp}$ & C & $12 \mathrm{bp}$ & $\mathrm{G}$ & $\mathrm{G}$ & G & G & A & $\mathrm{T}$ & G & G & G & C & $\mathrm{G}$ & C & C & G & G & $3 b p$ & $\mathrm{~T}$ \\
\hline A4 & A & A & $16 \mathrm{bp}$ & C & $12 \mathrm{bp}$ & $T$ & G & G & G & A & $\mathrm{T}$ & G & G & G & C & G & C & C & G & G & $3 b p$ & $\mathrm{~T}$ \\
\hline A5 & $A$ & $A$ & $16 \mathrm{bp}$ & C & $12 \mathrm{bp}$ & $T$ & G & G & G & A & $\mathrm{T}$ & G & G & G & $C$ & G & C & C & G & G & $3 b p$ & G \\
\hline A6 & A & $A$ & $16 \mathrm{bp}$ & C & $6 \mathrm{bp}$ & G & A & G & G & A & $\mathrm{T}$ & G & G & G & C & G & C & C & G & $G$ & $3 b p$ & $\mathrm{~T}$ \\
\hline A7 & A & $A$ & - & C & $12 \mathrm{bp}$ & G & G & G & G & A & $\mathrm{T}$ & G & G & G & C & G & C & C & G & G & $3 b p$ & $\mathrm{~T}$ \\
\hline A8 & G & A & - & $C$ & - & G & G & G & G & A & $\mathrm{T}$ & G & G & G & C & G & $C$ & C & G & G & $3 b p$ & $\mathrm{~T}$ \\
\hline A9 & A & A & - & C & - & G & G & G & G & A & $\mathrm{T}$ & G & G & G & A & G & C & A & G & G & $3 b p$ & G \\
\hline A10 & $A$ & $A$ & - & C & - & G & G & G & G & A & $\mathrm{T}$ & G & G & $G$ & $C$ & $G$ & C & C & $G$ & $G$ & $3 b p$ & G \\
\hline A11 & A & A & - & G & $6 \mathrm{bp}$ & G & A & G & $\mathrm{G}$ & A & $\mathrm{T}$ & G & G & $\mathrm{G}$ & C & G & C & C & $\mathrm{G}$ & G & $3 b p$ & $\mathrm{~T}$ \\
\hline $\mathrm{A} 12$ & $A$ & $A$ & - & C & $12 \mathrm{bp}$ & $\mathrm{G}$ & $\mathrm{G}$ & $\mathrm{G}$ & $\mathrm{G}$ & $A$ & $\mathrm{~T}$ & $\mathrm{G}$ & $\mathrm{G}$ & $\mathrm{G}$ & C & $\mathrm{G}$ & C & $C$ & $\mathrm{G}$ & $\mathrm{G}$ & $3 b p$ & G \\
\hline A13 & A & A & - & $C$ & $6 \mathrm{bp}$ & G & G & G & G & $A$ & $\mathrm{~T}$ & G & G & $\mathrm{G}$ & $C$ & G & $C$ & C & G & G & $3 b p$ & $\mathrm{~T}$ \\
\hline A14 & G & $A$ & - & G & $6 \mathrm{bp}$ & G & $A$ & $\mathrm{G}$ & G & $A$ & $\mathrm{~T}$ & G & $\mathrm{G}$ & $\mathrm{G}$ & C & G & $C$ & C & G & $\mathrm{G}$ & $3 b p$ & $\mathrm{~T}$ \\
\hline A15 & $G$ & A & - & C & $12 \mathrm{bp}$ & $T$ & G & G & G & A & $\mathrm{T}$ & G & $\mathrm{G}$ & G & C & G & C & C & $\mathrm{G}$ & G & $3 b p$ & $\mathrm{~T}$ \\
\hline A16 & $A$ & $A$ & - & $C$ & - & G & G & $\mathrm{G}$ & G & - & $\mathrm{T}$ & G & $\mathrm{G}$ & $\mathrm{G}$ & C & G & C & C & G & $\mathrm{G}$ & $3 b p$ & G \\
\hline A17 & A & A & $16 \mathrm{bp}$ & C & $12 \mathrm{bp}$ & $T$ & $\mathrm{G}$ & $\mathrm{G}$ & $\mathrm{G}$ & - & $\mathrm{T}$ & G & $\mathrm{G}$ & $\mathrm{G}$ & C & G & $C$ & C & G & $\mathrm{G}$ & $3 b p$ & G \\
\hline A18 & A & $A$ & $16 \mathrm{bp}$ & C & $12 \mathrm{bp}$ & $T$ & $G$ & $G$ & $\mathrm{G}$ & $A$ & $\mathrm{~T}$ & $\mathrm{G}$ & $G$ & $A$ & $C$ & $\mathrm{G}$ & C & C & $\mathrm{G}$ & $\mathrm{G}$ & $3 b p$ & G \\
\hline A19 & $A$ & $A$ & - & C & - & $\mathrm{G}$ & $\mathrm{G}$ & $\mathrm{G}$ & $\mathrm{G}$ & - & $\mathrm{T}$ & $\mathrm{G}$ & $\mathrm{G}$ & $\mathrm{G}$ & $A$ & G & $C$ & A & G & $\mathrm{G}$ & $3 b p$ & G \\
\hline A20 & A & A & - & C & - & G & G & $\mathrm{T}$ & C & A & C & C & - & G & A & $\mathrm{T}$ & $\mathrm{T}$ & A & C & $\mathrm{T}$ & $3 b p$ & G \\
\hline A21 & $A$ & $A$ & - & $C$ & - & G & G & G & G & $A$ & $\mathrm{~T}$ & G & $G$ & $\mathrm{G}$ & $A$ & $\mathrm{G}$ & C & A & $\mathrm{G}$ & G & - & G \\
\hline A22 & $A$ & $C$ & - & C & - & G & $\mathrm{G}$ & G & G & $A$ & $\mathrm{~T}$ & G & G & G & C & G & C & C & G & G & $3 b p$ & G \\
\hline
\end{tabular}


Table 3 Amino acid variations in the different alleles in O. sativa

\begin{tabular}{|c|c|c|c|c|c|c|c|c|c|}
\hline Haplotype & Frequency & 54 & 79 & 107 & 118 & 221 & 230 & 328 & 537 \\
\hline AP1 (A12) & $1.1 \%$ & $A$ & AAAA & A & $\mathrm{R}$ & EMV & $A$ & $\mathrm{~S}$ & K \\
\hline AP2 $(A 3, A 7)$ & $8.0 \%$ & $A$ & AAAA & A & $\mathrm{R}$ & EMV & $A$ & S & $\mathrm{N}$ \\
\hline AP3 (A5) & $14.9 \%$ & A & AAAA & s & $\mathrm{R}$ & EMV & A & S & K \\
\hline AP4 (A4, A15) & $3.4 \%$ & $A$ & AAAA & S & $\mathrm{R}$ & EMV & $A$ & S & $\mathrm{N}$ \\
\hline AP5 (A17) & $1.1 \%$ & A & AAAA & S & $\mathrm{R}$ & EM\& & & & \\
\hline AP6 (A13) & $0.6 \%$ & A & AA- & $A$ & $\mathrm{R}$ & EMV & A & S & N \\
\hline AP7 (A6) & $0.6 \%$ & A & AA- & A & $\mathrm{H}$ & EMV & A & S & $N$ \\
\hline $\operatorname{AP8}(A 9, A 10, A 22)$ & $32.0 \%$ & A & - - & A & $\mathrm{R}$ & EMV & A & S & K \\
\hline AP9 $(A 2, A 8)$ & $27.4 \%$ & $A$ & - - & A & $\mathrm{R}$ & EMV & A & S & $N$ \\
\hline $\operatorname{AP10}(\mathrm{A} 16, \mathrm{~A} 19)$ & $1.7 \%$ & A & -— & A & R & EM\& & & & \\
\hline $\operatorname{AP} 11(\mathrm{~A} 1, \mathrm{~A} 11, \mathrm{~A} 14)$ & $6.9 \%$ & G & AA- & A & $\mathrm{H}$ & EMV & A & $S$ & $N$ \\
\hline AP12 (A21) & $1.1 \%$ & A & - & A & $R$ & EMV & A & - & K \\
\hline AP13 (A20) & $0.6 \%$ & A & -— & A & R & YT\& & & & \\
\hline AP14 (A18) & $0.6 \%$ & A & AAAA & S & $\mathrm{R}$ & EMV & T & S & K \\
\hline
\end{tabular}

The number in the first line indicated the variable amino acid sites of the GN1A protein; the Gn1a alleles are in brackets

indels at $-663 \mathrm{bp}$ and $-701 \mathrm{bp}$, as well as a 16-bp deletion in the 5'UTR (Fig. 3a). Based on these three indels, five combinations named Promoter- 1 to Promoter- 5 were detected, and the frequency of Promoter-1 (24.12 \%) and Promoter-4 (64.17 \%) was apparently higher than the other alleles (Fig. 3b). Promoter-1 contained 6 alleles, of which allele AP3 was the major allele, accounting for $63.41 \%$. Promoter-4 was comprised of 8 alleles, among which AP8 and AP9 were the most frequent alleles and accounted for $50.93 \%$ and $36.11 \%$. The other alleles were less than $6 \%$.

AP3, AP8 and AP9 were the popular alleles in rice populations, which indicated that Promoter-1 and Promoter-4 were the two major types of promoters in the cultivars. Then, we assayed the expression level of Gnla between Promoter-1 and Promoter-4 in young leaves and inflorescences in the cultivars. The qRT-PCR

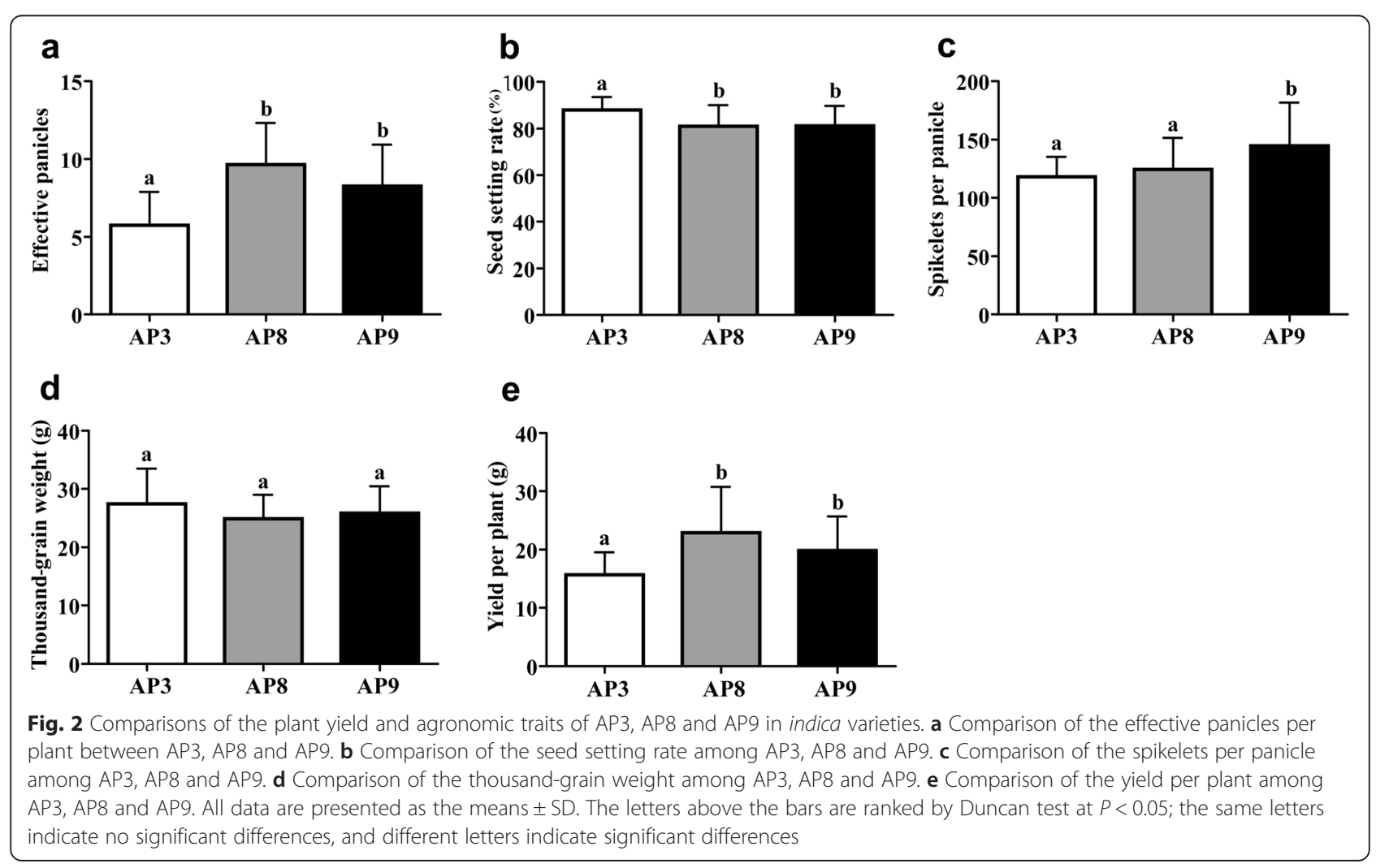


a

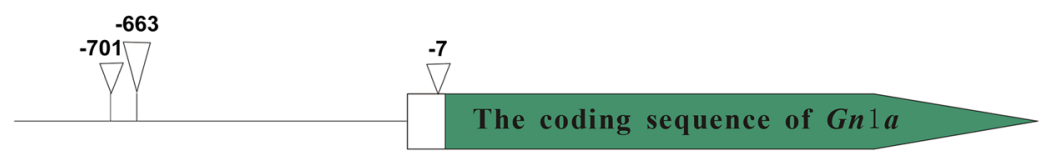

b

\begin{tabular}{|c|c|c|c|c|c|}
\hline & -701 & -663 & -7 & Alleles frequency in the different promoter & $\begin{array}{l}\text { Frequency in } \\
\text { cultivars }\end{array}$ \\
\hline Promoter-1 & (CTAT) 2 & $(\mathrm{GGAA}) 2$ & $16 \mathrm{bp}$ & $\begin{array}{l}\operatorname{AP2}(12.2 \%), \operatorname{AP} 3(63.41 \%), \operatorname{AP} 5(4.88 \%), \operatorname{AP9}(4.88 \%), \\
\operatorname{AP} 11(12.2 \%), \operatorname{AP} 14(2.44 \%)\end{array}$ & $24.12 \%$ \\
\hline Promoter-2 & $($ CTAT $) 1$ & $(\mathrm{GGAA}) 2$ & $16 \mathrm{bp}$ & $\operatorname{AP2}(10 \%), \operatorname{AP} 4(50 \%), \operatorname{AP7}(10 \%), \operatorname{AP} 9(20 \%), \operatorname{AP} 11(10 \%)$ & $5.88 \%$ \\
\hline Promoter-3 & (CTAT) 2 & $(\mathrm{GGAA}) 2$ & - & AP2 (66.67\%), AP8 (33.33\%) & $0.59 \%$ \\
\hline Promoter-4 & (CTAT) 1 & $(\mathrm{GGAA}) 2$ & - & $\begin{array}{l}\operatorname{AP} 4(0.93 \%), \operatorname{AP} 6(0.93 \%), \operatorname{AP} 8(50.93 \%), \operatorname{AP} 9(36.11 \%) \\
\operatorname{AP} 10(2.78 \%), \operatorname{AP} 11(5.56 \%), \operatorname{AP} 12(1.85 \%), \operatorname{AP} 13(0.93 \%)\end{array}$ & $64.71 \%$ \\
\hline Promoter -5 & (CTAT) 1 & $(\mathrm{GGAA}) 1$ & - & $\operatorname{AP} 1(25 \%), \quad A P 3(75 \%)$ & 4. $71 \%$ \\
\hline
\end{tabular}

\section{C}

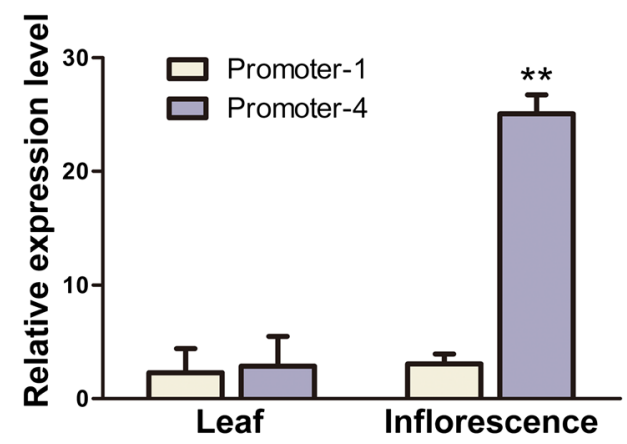

d

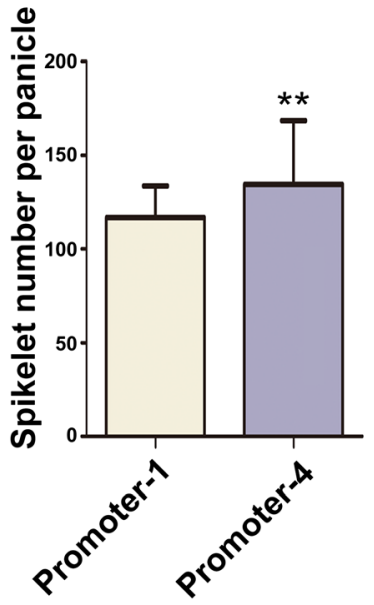

Fig. 3 The structure and transcription level of the different promoters. a The variation sites where the different deletions occur; " $\nabla$ " indicates the deletions. b Different types of promoters; "-" indicates a 16-bp deletion. The percentage in brackets located after the alleles indicates the frequency of the alleles in the corresponding promoters. $\mathbf{c}$ Relative expression level between Promoter-1 and Promoter-4 in young leave and inflorescences. "**" indicates a significance difference $(P<0.01)$. d Comparison of the number of spikelets per panicle between Promoter-1 and Promoter-4. "**" indicates a significance difference $(P<0.01)$

analysis showed that there was no significant difference in either promoter in the young leaves, but the expression level of Promoter-4 was 20-fold higher than that of Promoter-1 in the inflorescences (Fig. 3c). Furthermore, the number of spikelets per panicle between Promoter-1 and Promoter-4 were analyzed, and the results indicated that Promoter- 4 generated more spikelets than Promoter1 (Fig. 3d). These results suggested that the protein sequence and expression level of Gn1a might affect the number of grains, as cultivars harboring AP9 could produce more grains than cultivars with AP8.

\section{Distribution and Evolutionary Pattern of the GN1A Alleles in the Oryza Genus}

To understand the general status of the GN1A alleles in the Oryza genus, we further investigated the distribution of the GN1A alleles in the wild rice O. rufipogon, and found that, with the exception of AP1, AP3 and AP8, nine new alleles, named AP15 to AP23, were detected in O. rufipogon (Additional file 3: Table S3). Of these, 7 out of 21 wild rice accessions harbored AP1, which indicated that AP1 might be the original allele in the Oryza genus.

A phylogenetic network for alleles of GN1A was constructed based on the amino acid sequence of the 23 alleles in O. sativa and O. rufipogon (Fig. 4). Using this network, we found that AP3, AP8 and AP9 were the most popular alleles in the cultivars. Interestingly, AP8 and AP9 were primarily distributed in indica, and rarely found in japonica rice. However, the ratios of AP3, AP5, AP10 and AP15 were not apparently different between indica and japonica, which reflected a strong artificial selection during rice domestication. Actually, there was 


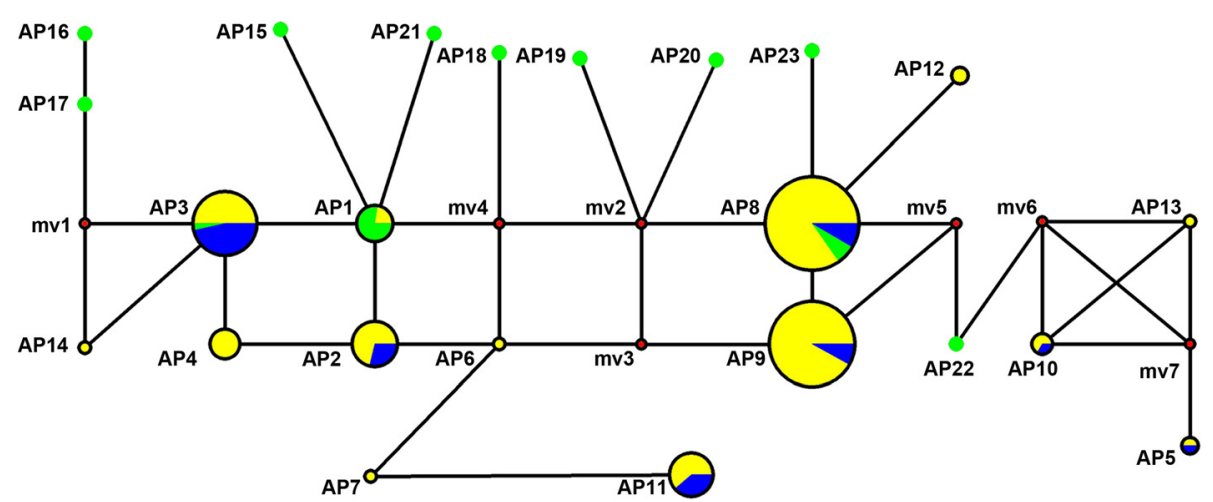

Fig. 4 The phylogenetic network for the 23 alleles of GN1A between wild and cultivated rice species. Each circle represents an allele. The size of the circles indicates the frequency of each allele. The green, yellow and blue circles indicate the allelic distribution in the wild rice, indica and japonica species, respectively. The red median vectors (mv) represent a hypothesized sequence that is required to connect the existing sequences within the network with maximum parsimony. The solid lines represent one mutation step that interconnects adjacent alleles

only a single amino acid variation at position 537 between AP8 and AP9 (Table 3), implying their tight evolutionary relationship.

Based on the frequency and distribution of the alleles in the cultivars and O. rufipogon, we assumed that AP1 might be the original allele, as it had the highest frequency in wild rice. Thus, we proposed a schematic diagram to describe the possible evolutionary pattern of the main alleles of Gn1a (Fig. 5). The model suggested that in the O. rufipogon population, the primitive allele AP1 might have evolved to AP3 and AP8, respectively, by the loss of two or three alanine repeats at the amino acid 79 , as the majority of wild rice encoded the amino acid 79 by four-alanine repeats. Then, the lysine 537 of AP1 and AP8 had mutated to asparagine in AP2 and AP9, respectively. AP9 was then strongly selected during domestication and ultimately became the most prevalent allele indifferent rice populations because of its larger panicle.

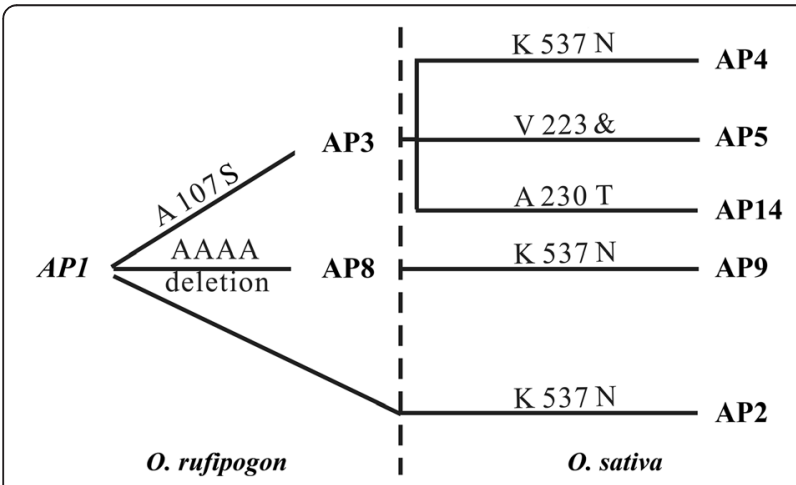

Fig. 5 Evolutionary pattern of the most frequent alleles of GN1A in the Oryza genus. Substitution and deletion events occurred above each line, and the substitution of $\mathrm{K} 537$ to $\mathrm{N}$ played an important role in the evolutionary process from wild rice to the common cultivars

\section{The Artificial Selection Pattern of Gn1a}

The Tajim's D (Tajima 1989), Fu and Li's D and F tests (Fu 1997) are usually employed to assess the trends of artificial selection of genes in plant populations. To understand whether the evolutionary process of Gn1a was affected by artificial selection, we investigated the selection pattern using the Tajima's D, Fu and Li's D and $\mathrm{F}$ tests in the different rice species (Table 1). The values of Fu and Li's D and F tests were -3.34715 and -2.86108 in $O$. sativa, which were significantly negative values compared to O. rufipogon. This result indicated that the excess low-frequency polymorphisms of the alleles in $O$. sativa deviated from the neutral expectation, implying there was a strong artificial selection of Gn1a in cultivar populations (Du et al. 2011).

Then we investigated the alleles of GN1A in the pedigree of Guichao 2, a high grain-yield cultivar bred in China in the 1980s-1990s, as a model to test how artificial selection influences the distribution of the GN1A alleles in breeding programs. In this pedigree, all of the tested accessions carried allele AP9, with the exception of Qingnong'ai (Additional file 4: Figure S1). This result implied that when another allele was introduced in the pedigree, the excellent AP9 allele was still selected in the offspring, namely artificial selection accelerated the concentration of the large panicle AP9 allele of GN1A in cultivar populations. This was consistent with the Tajim's $\mathrm{D}, \mathrm{Fu}$ and Li's $\mathrm{D}$ and $\mathrm{F}$ tests, and provided us a new insight into the evolutionary pattern of GN1A during rice domestication. These high-yield-related alleles could facilitate the development of high-yield rice in future breeding programs.

\section{Geographic Distribution of the GN1A Alleles}

We analyzed the regional distribution of the GN1A alleles in the cultivars to explore the relationship 
between the evolutionary pattern and geographic distribution of the GN1A alleles (Fig. 6). Interestingly, these alleles showed a strong distinctive geographic character; AP3, AP8 and AP9 spread over seven countries in different continents. AP3 was primarily located in Brazil, AP8 was primarily located in Indonesia and the Philippines, and AP9 was primarily concentrated in China. The other alleles were mostly limited to a single area; for example AP6, AP7, AP13 and AP14 were found only in Thailand, India, the Philippines and Colombia, respectively. These results indicated that AP3 had primarily concentrated in the Americas, and AP8 and AP9 were the most frequent alleles in Asia.

\section{Discussion}

Plant hormones, including auxin (Wang et al. 2014; Zhang et al. 2015a; Zhao et al. 2015), gibberellins (Asano et al. 2011; Wu et al. 2014; Zhang et al. 2015b), abscisic acid (Santiago et al. 2009; Zhang et al. 2015b), ethylene (Strader et al. 2010; Yin et al. 2015), cytokinin (Ashikari et al. 2005; Bartrina et al. 2011; Li et al. 2013) and brassinosteroids (Kim et al. 2012; Sun et al. 2015), are important for plant growth and development. Cytokinin has been considered to be an important factor for modulating inflorescence meristem development (Nishimura et al. 2004; Riefler et al. 2006), and, hence, plays a crucial role in determining the rice grain yield. Gn1a, which encodes cytokinin oxidase/dehydrogenase, plays a critical role in regulating the number of grains per panicle and rice yield. It has been verified that pyramid Gn1a and $s d 1$ could increase the grain number by $23 \%$, without changing the grain size (Ashikari et al. 2005). It will be important to explore the high-yield-related alleles of Gn1a in breeding programs as effective strategy to create highyield rice to satisfy the daily requirements of the increasing global population.

In this study, we analyzed the sequences of GN1A alleles in cultivars and common wild rice accessions, and found that AP3, AP8 and AP9 were the most frequent alleles in the cultivars. Of these, AP9 was superior to AP3 and AP8 with regard to the number of spikelets. Compared to AP3, both AP8 and AP9 had a four-alanine deletion in the first exon of GN1A. However, there was no significant difference in the number of spikelets between AP3 and AP8, which

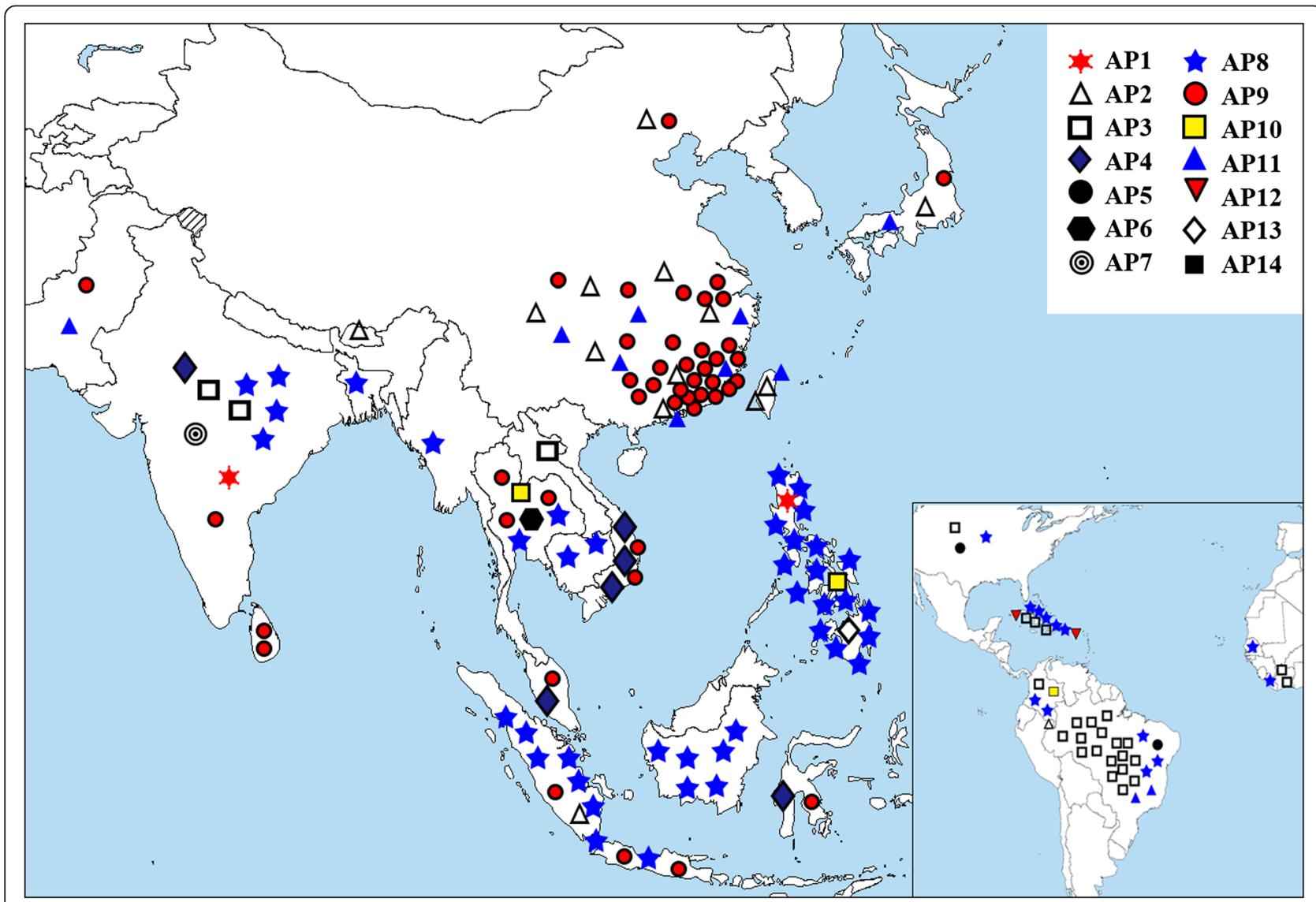

Fig. 6 Geographic distribution of the 14 different alleles of GN1A. Symbols with different colors indicate different alleles, and each symbol represents one accession 
indicated that these four tandem alanine repeats in the first exon did not have a special function in spikelet development. When lysine 537 in AP8 was substituted to asparagine in AP9, the spikelets per panicle increased. Thus, we presumed that the substitution of amino acid 537 in GN1A exerted a critical function on determining the grains number of rice panicles. Although AP9 promoted an increased number of spikelets, the grain yield for the AP9 allele did not increase significantly compared to AP8. It was possibly that other genes related to effective panicles and grain weight might compromise the effects of AP9 in a complicated genetic background. To accurately understand the effects and the molecular mechanism of AP8 and AP9 on rice productivity, a set of nearly isogenic lines containing each of these GN1A alleles should be constructed to exclude the background noise in rice breeding programs.

Compared to the other alleles, AP1 was the most frequently detected allele in the common wild rice accessions in this study, and the frequency reached $33.3 \%$ in O. rufipogon. This result implied that AP1 was likely the original allele in O. rufipogon. Based on the protein sequence, we deduced that both AP3 and AP8 might originate from AP1 because there was only one variant site between AP1 and AP3/AP8. Interestingly, there was only one amino acid difference between AP8 and AP9, which substituted lysine 537 in AP8 and for asparagines 537 in AP9. Because asparagines 537 was not detected in O. rufipogon, we proposed that AP9 was directly mutated from AP8 in the cultivars. AP3 might further differentiate into other alleles, such as AP4, AP5 and AP14. However, it was still difficult for us to deduce the original ancestor of the other rare alleles in the rice accessions because of the limited wild rice accession samples investigated in this study. More wild rice accessions in the Oryza genus need to be analyzed to elucidate the detailed evolutionary pattern of the GN1A alleles.

\section{Conclusions}

In this study, we characterized allele AP9 as the most frequent allele, due to the large panicle and high yield per plant, which may facilitate the development of highyield rice in future breeding programs.

\section{Methods}

\section{Plant Materials and Trait Analysis}

The 175 cultivar lines and 21 wild rice accessions of O. rufipogon, from 19 countries representing a diverse range of grains number and plant yield, were collected from the International Rice Research Institute (IRRI) and our laboratory were grown under normal field conditions on the island of Hainan, China and the E'Zhou experiment station in E'Zhou (Hubei Province, China) from 2012 to 2014. Most cultivars are landraces from rice-growing regions in China, Indonesia and the Philippines. Ten plants with a density of $16.5 \mathrm{~cm} \times$ $26.5 \mathrm{~cm}$ were planted in a row and 5 rows of each accession were planted.

The number of effective panicles per plant was counted as the number of tillers bearing more than 5 grains. The number of spikelets per panicle was measured as the total number of spikelets divided by the effect panicles. The yield per plant was scored as the total weight of the grains per plant. The thousand-grain weight was scored as the yield per plant divided by the total number of grains multiplied by 1000 . The seed setting rate was tested as the number of grains per panicle divided by the the number of spikelets per panicle. All traits were measured from 5 randomly sampled plants for each accession after harvest.

\section{DNA Extraction, PCR Amplification and Sequencing}

The genomic DNA was extracted from fresh leaves using cetyltrimethyl ammonium bromide (Murray and Thompson 1980). The primers that were used to amplify the promoter, 5'UTR and CDS were designed using the Gn1a allele of Nipponbare. The PCRs were performed under the standard PCR protocols, and the $20 \mu \mathrm{l}$ PCR system contained $50 \mathrm{ng}$ of the genomic DNA template, $0.5 \mu \mathrm{l}$ of the forward and reverse primers (both $10 \mu \mathrm{M}), 1.6 \mu \mathrm{l}$ of $2.5 \mathrm{mM}$ dNTPs, $1.6 \mu \mathrm{l}$ of $25 \mathrm{mM}$ $\mathrm{MgCl}_{2}, 0.3 \mu \mathrm{l}$ of $5 \mathrm{U} / \mu \mathrm{lrTap}$ polymerase and $2 \mu \mathrm{l}$ $10 \times$ rTap buffer. The $20 \mu \mathrm{l}$ PCR products were recycled using a TIANGEN recycling kit, and then ligated into the pGEM18-T Easy Vector. Each independent plasmid DNA was sequenced by Tsingke (Wuhan, China). The correct DNA sequence was selected after comparing three plasmids from each accession. All primers used for PCR amplification were presented in Additional file 5: Table S4.

\section{DNA Sequences Analysis and Alleles Analysis}

The DNA sequences from the different Oryza accessions were aligned using CLUSTAL W version 2.0 and adjusted manually with BIOEDIT. Two measures of nucleotide variability, the average number of nucleotide differences per site $(\pi)$ (Beverley et al. 1987) and nucleotide diversity based on the proportion of segregating sites $(\theta \omega)$ (Watterson 1975), were obtained using DNASP v5.0 (Librado and Rozas 2009). The selection tests, including Tajima's D test (Tajima 1989), Fu and Li's D and F tests, were performed using DNASP v5.0. The sliding-window method was employed to value the polymorphisms across the coding sequence of Gn1a, using a window size of 100 and a step size of 20, in which pairwise insertions and deletions were removed via DNASP v5.0. The allelic diversity analysis 
was performed using BIOEDIT and the MAGE 5.0 program (Tamura et al. 2011). The phylogenetic network was constructed by Network 4.611 according to the corresponding user guide.

\section{RNA Isolation and RT-PCR}

The total RNA was prepared from the fresh leaves at the seedling stage and inflorescences at the young panicle stage using an RNAprep pure Plant Kit (Invitrogen, Carlsbad, CA, USA). The first-strand cDNA was synthesized from the total RNA using a Superscript II RT Kit (Invitrogen, Carlsbad, CA, USA). All RT-PCRs were performed using the first-strand cDNA as the template; 35 PCR cycles were used to amplify the Gn1a transcripts, and 25 PCR cycles were used to amplify the Actin transcripts. Real-time quantitative PCR was performed in the Roche Light Cycler 480 PCR system according to the manufacturer's instruction. The rice Actin gene was used as the internal control, and three technical replicates were performed for each sample. The expression level was calculated using the relative quantification method.

\section{Statistical Analysis}

The statistical analyses were performed using the SPSS software, and the comparisons of the number of spikelets per panicle, thousand-grain weight, seed setting rate, effective panicles and plant yield among the alleles were calculated by one-way ANOVA. Nonparametric tests were performed if there was no homogeneity of variance. If the results of the analysis were significant $(P<0.05)$, Duncan's multiple range test was used as a post hoc test for multiple comparisons. The histograms were constructed using the GraphPad Prism 5 software and modified by Adobe Photoshop CS5. All tests were performed at least three times.

\section{Additional files}

Additional file 1: Table S1. Germplasm variety information and their alleles of Gn1a. "NO" in the first line indicates the serial number in the field; SPP, TGW, PY, SSR and EP indicate the number of spikelets per panicle, thousand-grain weight, seed setting rate and effective panicles per plant, respectively; "/" indicates that the data were not available. (DOC 266 KB)

Additional file 2: Table S2. Basic information on the wild rice varieties. The number ahead of each material indicated the serial number in the field in 2012, and the alleles were established based on the different amino acid sequences among the wild rice varieties. (DOC $38 \mathrm{~KB}$ )

Additional file 3: Table S3. Amino acid variations of the different alleles in $O$. rufipogon. The number in the first line indicates the variable amino acid sites of the GN1A protein and the GN1A alleles are in brackets; "-" indicates the deletion of the corresponding amino acid, and " $\&$ " indicates the stop codon of the protein. (DOC $68 \mathrm{~kb}$ )
Additional file 4: Figure S1. Allele analysis of the pedigree of Guichao 2. The colored materials were selected for this study; the green varieties harbored the AP9 allele and the yellow varieties contained the AP2 allele. (DOC $157 \mathrm{~kb}$ )

Additional file 5: Table S4. Primers used in this study. (DOC $30 \mathrm{~kb}$ )

\section{Competing interests}

The authors declare that they have no competing interests.

\section{Authors' contributions}

LS and ZY designed the study, WJ performed the entire experiment, $\mathrm{XH}$ extracted and analyzed the DNA sequences of the rice materials, LN participated in field management, FF, WL and WJ investigated the yield traits of the materials, and WJ performed the statistical analyses and wrote the paper. All authors read and approved the final manuscript.

\section{Acknowledgments}

This research was partially supported by the 973 Program (2011CB100102), the 863 program (2014AA10A604-9), and the Natural Science Foundation (31370363) of China.

Received: 4 July 2015 Accepted: 1 December 2015

Published online: 16 December 2015

\section{References}

Asano K, Yamasaki M, Takuno S, Miura K, Katagiri S, Ito T, Doi K, Wu J, Ebana K, Matsumoto T, Innan H, Kitano H, Ashikari M, Matsuoka M (2011) Artificial selection for a green revolution gene during japonica rice domestication. Proc Natl Acad Sci U S A 108(27):11034-11039. doi:10.1073/pnas.1019490108

Ashikari M, Sakakibara H, Lin S, Yamamoto T, Takashi T, Nishimura A, Angeles ER, Qian Q, Kitano H, Matsuoka M (2005) Cytokinin oxidase regulates rice grain production. Science 309(5735):741-745. doi:10.1126/science.1113373

Bartrina I, Otto E, Strnad M, Werner T, Schmulling T (2011) Cytokinin regulates the activity of reproductive meristems, flower organ size, ovule formation, and thus seed yield in Arabidopsis thaliana. Plant Cell 23(1):69-80. doi:10.1105/ tpc. 110.079079

Beverley SM, Ismach RB, Pratt DM (1987) Evolution of the genus Leishmania as revealed by comparisons of nuclear DNA restriction fragment patterns. Proc Natl Acad Sci U S A 84(2):484-488

Dong X, Wang X, Zhang L, Yang Z, Xin X, Wu S, Sun C, Liu J, Yang J, Luo X (2013) Identification and characterization of OSEBS, a gene involved in enhanced plant biomass and spikelet number in rice. Plant Biotechnol J. 11(9):10441057. doi:10.1111/pbi.12097

Du H, Ouyang Y, Zhang C, Zhang Q (2011) Complex evolution of S5, a major reproductive barrier regulator, in the cultivated rice Oryza sativa and its wild relatives. New Phytol 191(1):275-287. doi:10.1111/j.1469-8137.2011.03691.x

Fu YX (1997) Statistical tests of neutrality of mutations against population growth, hitchhiking and background selection. Genetics 147(2):915-925

Huang X, Qian Q, Liu Z, Sun H, He S, Luo D, Xia G, Chu C, Li J, Fu X (2009) Natural variation at the DEP1 locus enhances grain yield in rice. Nat Genet 41(4):494-497. doi:10.1038/ng.352

Ikeda K, Ito M, Nagasawa N, Kyozuka J, Nagato Y (2007) Rice ABERRANT PANICLE ORGANIZATION 1, encoding an F-box protein, regulates meristem fate. Plant J 51(6):1030-1040. doi:10.1111/j.1365-313X.2007.03200.x

Jiao Y, Wang Y, Xue D, Wang J, Yan M, Liu G, Dong G, Zeng D, Lu Z, Zhu X, Qian Q, Li J (2010) Regulation of OSSPL14 by OsmiR156 defines ideal plant architecture in rice. Nat Genet 42(6):541-544. doi:10.1038/ng.591

Kim TW, Michniewicz M, Bergmann DC, Wang ZY (2012) Brassinosteroid regulates stomatal development by GSK3-mediated inhibition of a MAPK pathway. Nature 482(7385):419-422. doi:10.1038/nature10794

Li S, Zhao B, Yuan D, Duan M, Qian Q, Tang L, Wang B, Liu X, Zhang J, Wang J, Sun J, Liu Z, Feng YQ, Yuan L, Li C (2013) Rice zinc finger protein DST enhances grain production through controlling Gn1a/OsCKX2 expression. Proc Natl Acad Sci U S A 110(8):3167-3172. doi:10.1073/pnas.1300359110

Librado P, Rozas J (2009) DnaSP v5: a software for comprehensive analysis of DNA polymorphism data. Bioinformatics 25(11):1451-1452. doi:10.1093/ bioinformatics/btp187

Ma X, Cheng Z, Qin R, Qiu Y, Heng Y, Yang H, Ren Y, Wang X, Bi J, Zhang X, Wang J, Lei C, Guo X, Wu F, Jiang L, Wang H, Wan J (2012) OsARG encodes an arginase 
that plays critical roles in panicle development and grain production in rice. Plant J. 73(2):190-200. doi:10.1111/j.1365-313X. 2012.05122.x

Miura K, Ikeda M, Matsubara A, Song XJ, Ito M, Asano K, Matsuoka M, Kitano H, Ashikari M (2010) OSSPL14 promotes panicle branching and higher grain productivity in rice. Nat Genet 42(6):545-549. doi:10.1038/ng.592

Murray MG, Thompson WF (1980) Rapid isolation of high molecular weight plant DNA. Nucleic Acids Res 8(19):4321-4325

Nishimura C, Ohashi Y, Sato S, Kato T, Tabata S, Ueguchi C (2004) Histidine kinase homologs that act as cytokinin receptors possess overlapping functions in the regulation of shoot and root growth in Arabidopsis. Plant Cell 16(6): 1365-1377. doi:10.1105/tpc.021477

Riefler M, Novak O, Strnad M, Schmulling T (2006) Arabidopsis cytokinin receptor mutants reveal functions in shoot growth, leaf senescence, seed size, germination, root development, and cytokinin metabolism. Plant Cell 18(1): 40-54. doi:10.1105/tpc.105.037796

Santiago J, Dupeux F, Round A, Antoni R, Park SY, Jamin M, Cutler SR, Rodriguez PL, Marquez JA (2009) The abscisic acid receptor PYR1 in complex with abscisic acid. Nature 462(7273):665-668. doi:10.1038/nature08591

Strader LC, Chen GL, Bartel B (2010) Ethylene directs auxin to control root cell expansion. Plant J 64(5):874-884. doi:10.1111/j.1365-313X.2010.04373.X

Sun S, Chen D, Li X, Qiao S, Shi C, Li C, Shen H, Wang X (2015) Brassinosteroid signaling regulates leaf erectness in Oryza sativa via the control of a specific U-type cyclin and cell proliferation. Dev Cell 34(2):220-228. doi:10.1016/j. devcel.2015.05.019

Tajima F (1989) Statistical method for testing the neutral mutation hypothesis by DNA polymorphism. Genetics 123(3):585-595

Tamura K, Peterson D, Peterson N, Stecher G, Nei M, Kumar S (2011) MEGA5: molecular evolutionary genetics analysis using maximum likelihood, evolutionary distance, and maximum parsimony methods. Mol Biol Evol 28(10):2731-2739. doi:10.1093/molbev/msr121

Wang S, Zhang S, Sun C, Xu Y, Chen Y, Yu C, Qian Q, Jiang DA, Qi Y (2014) Auxin response factor (OSARF12), a novel regulator for phosphate homeostasis in rice (Oryza sativa). New Phytol 201(1):91-103. doi:10.1111/nph.12499

Watterson GA (1975) On the number of segregating sites in genetical models without recombination. Theor Popul Biol 7(2):256-276

Weng J, Gu S, Wan X, Gao H, Guo T, Su N, Lei C, Zhang X, Cheng Z, Guo X, Wang J, Jiang L, Zhai H, Wan J (2008) Isolation and initial characterization of GW5, a major QTL associated with rice grain width and weight. Cell Res 18(12):11991209. doi:10.1038/cr.2008.307

Wu J, Zhu C, Pang J, Zhang X, Yang C, Xia G, Tian Y, He C (2014) OsLOL1, a C2C2-type zinc finger protein, interacts with OsbZIP58 to promote seed germination through the modulation of gibberellin biosynthesis in Oryza sativa. Plant J 80(6):1118-1130. doi:10.1111/tpj.12714

Xing Y, Zhang Q (2010) Genetic and molecular bases of rice yield. Annu Rev Plant Biol 61:421-442. doi:10.1146/annurev-arplant-042809-112209

Yin CC, Ma B, Collinge DP, Pogson BJ, He SJ, Xiong Q, Duan KX, Chen H, Yang C, Lu X, Wang YQ, Zhang WK, Chu CC, Sun XH, Fang S, Chu JF, Lu TG, Chen SY, Zhang JS (2015) Ethylene responses in rice roots and coleoptiles are differentially regulated by a carotenoid isomerase-mediated abscisic acid pathway. Plant Cell 27(4):1061-1081. doi:10.1105/tpc.15.00080

Yoshida A, Sasao M, Yasuno N, Takagi K, Daimon Y, Chen R, Yamazaki R, Tokunaga H, Kitaguchi Y, Sato Y, Nagamura Y, Ushijima T, Kumamaru T, lida S, Maekawa M, Kyozuka J (2013) TAWAWA1, a regulator of rice inflorescence architecture, functions through the suppression of meristem phase transition. Proc Natl Acad Sci U S A 110(2):767-772. doi:10.1073/pnas.1216151110

Zhang S, Wang S, Xu Y, Yu C, Shen C, Qian Q, Geisler M, de Jiang A, Qi Y (2015a) The auxin response factor, OsARF19, controls rice leaf angles through positively regulating OsGH3-5 and OsBRI1. Plant Cell Environ 38(4):638-654. doi:10.1111/pce.12397

Zhang Y, Lan H, Shao Q, Wang R, Chen H, Tang H, Zhang H, Huang J (2015b) An A20/AN1-type zinc finger protein modulates gibberellins and abscisic acid contents and increases sensitivity to abiotic stress in rice (Oryza sativa L.). J Exp Bot. doi:10.1093/jxb/erv464

Zhao L, Tan L, Zhu Z, Xiao L, Xie D, Sun C (2015) PAY1 improves plant architecture and enhances grain yield in rice. Plant J 83(3):528-536. doi:10 $1111 /$ tpj.12905

\section{Submit your manuscript to a SpringerOpen ${ }^{\odot}$ journal and benefit from:}

- Convenient online submission

- Rigorous peer review

- Immediate publication on acceptance

- Open access: articles freely available online

- High visibility within the field

- Retaining the copyright to your article

Submit your next manuscript at $\gg$ springeropen.com 